\title{
\#SupportTheCause: Identifying Motivations to Participate in Online Health Campaigns
}

\author{
Dong Nguyen $^{1} \quad$ Tijs van den Broek ${ }^{2}$ \\ Claudia Hauff $^{3} \quad$ Djoerd Hiemstra $^{4}$ Michel Ehrenhard $^{2}$ \\ ${ }^{1}$ Human Media Interaction, University of Twente, d. nguyen@utwente. n I \\ ${ }^{2}$ NIKOS, University of Twente, $\{t . a \cdot v a n d e n b r o e k, m .1 . e h r e n h a r d\} @ a t w e n t e . n I$ \\ ${ }^{3}$ Web Information Systems, Delft University of Technology, c . hauffetudelft.n I \\ ${ }^{4}$ Database Group, University of Twente, d.hiemstra@utwente.nl
}

\begin{abstract}
We consider the task of automatically identifying participants' motivations in the public health campaign Movember and investigate the impact of the different motivations on the amount of campaign donations raised. Our classification scheme is based on the Social Identity Model of Collective Action (van Zomeren et al., 2008). We find that automatic classification based on Movember profiles is fairly accurate, while automatic classification based on tweets is challenging. Using our classifier, we find a strong relation between types of motivations and donations. Our study is a first step towards scaling-up collective action research methods.
\end{abstract}

\section{Introduction}

Social media is a valuable source for studying health-related behaviors (De Choudhury, 2014). For example, Twitter was used for disease surveillance (Lamb et al., 2013; Aramaki et al., 2011), and was studied for its role in disseminating medical information (Desai et al., 2012) and organizing public health campaigns (Emery et al., 2014; Wehner et al., 2014). Social media data provides many opportunities to study social phenomena such as health campaigns, but statistics based on aggregating across social media users only provide a big picture of the phenomenon. A deeper analysis of such phenomena requires fine-grained information about the involved users. Since such information is often not readily available, numerous studies have appeared on automatically inferring user characteristics (Bamman et al., 2014; Eisenstein et al., 2010; Nguyen et al., 2013).

In the context of health campaigns, social scientists have been interested in the motivations of the participants (Cugelman et al., 2011). Knowledge about individual motivations helps to explain the emergence and effectiveness of collective action, such as volunteering (Bekkers and Wiepking, 2011) or mobilizing other people (van den Broek et al., 2015). The Social Identity Model of Collective Action (SIMCA) (van Zomeren et al., 2008) identifies three key motivations of participants: 1) social identification with the campaign organization and community, 2) a perception of injustice about the cause, and 3) collective efficacy, the collective belief that the campaign can make a difference. Taken together, these three motivations predict the chance that an individual will participate in collective action, such as participation in an online health campaign. Aggregating motivations to group-level may explain the effectiveness of online health campaigns. Social scientists, however, have not used computational methods to measure these motivations (Johnston et al., 2009), so that their analyses are often confined to small datasets.

Our study is a first step towards scaling-up collective action research methods. To do so, we explore automatic classification of the motivation types according to the SIMCA model. We analyze the global health campaign Movember (movember.com), which aims to raise funds and awareness of men-related health issues by engaging online conversations. Movember's fundraisers ask their friends to sponsor their moustache and their efforts in the month of November. The funds are donated to research concerned with menrelated health issues, such as prostate cancer.

Movember participants provide their motivations in their Movember profile. For example, a participant writing 'In honor of my Grandfather' could be considered having an injustice motivation, while 'To lead the brave men of Team [...] (and our exceptionally understanding significant others) in epic moustachery.' indicates a social identification motivation. Because such explicit motivation statements are not available for many online health campaigns, we also explore motiva- 
tion classification based on the tweets of participants during the campaign instead.

Our paper makes the following contributions:

- We automatically classify the motivations of Movember participants and explore the use of free-text motivations provided in Movember profiles and tweets posted by the participants during the campaign (Section 3 ).

- We apply our classifier to all $U S$ and $U K$ Movember profiles and find that participants with an injustice motivation raise significantly more funds (Section 4).

\section{Dataset}

In this section we discuss the collection and the annotation of the data.

\subsection{Collection}

We collect data from two different sources.

Movember Profiles We focus on participants from the two countries with the highest number of English speaking Movember participants: the United States and the United Kingdom. From Movember we obtained the identifiers of all participants of these two countries and we crawled all US and UK Movember profiles in May 2015. We extracted information such as the name, motivation (free-text), amount raised and whether the participant was part of a team. We collected 166,422 US and 138,546 UK profiles.

Twitter Data We link Movember participants to Twitter accounts based on tweets with a link to a Movember profile in 2013 and 2014 (e.g., 'please support my moustache [LINK]'). If the Levenshtein distance between the name of the author of the tweet and the name in the Movember profile was 1 or less, we considered it a match (in total: 5,519 users). Manual inspection of 100 matches showed that this method was highly precise (100\% precision). However, some matches were missed due to the low Levenshtein distance threshold. For each Twitter user in our dataset, we collected the last 3,600 tweets.

We kept all tweets written between October 18 and December 14 (2 weeks before and after the campaign). For each user, we used tweets from either 2013 or 2014, depending on whether the user posted a tweet with a Movember link at least once during the period, given preference to the year 2014.

\subsection{Annotation}

We annotated the campaign participants based on their provided motivations in their 'My motivation' section of their Movember profiles. The motivation categories in our codebook are based on the Social Identity Model of Collective Action (van Zomeren et al., 2008):

- Injustice: A shared emotion that includes both affective (e.g, anger) and cognitive perceptions (ideology) of an unfair situation (van Zomeren et al., 2008). It covers the ideological motivation to join a campaign, when potential participants compare the cause and the situation of patients with their personal values (Klandermans, 2004). For example, 'my dad', 'I had testicular cancer' or 'because men's health is important to me'.

- Social identity: A sense of belonging together that emerges out of common attributes, experiences and external labels (van Zomeren et al., 2008). Participants may have social motivations to identify with the online health campaign, while not being interested in the cause (Kristofferson et al., 2014). This category includes psychological benefits, such as reputation or fun, that the social interactions of a campaign provide. For example, 'my friends asked me again to join them', or 'a great excuse to grow a stache'.

- Collective efficacy: The shared belief that ones group is capable of resolving its grievances through a campaign (Bandura, 2000; Klandermans, 2004; van Zomeren et al., 2008), for example by stating 'this campaign can make a difference!'.

Multiple motivations may be assigned to a single campaign participant. Exactly recurring motivation texts that occurred frequently (more than 50 times, based on data analysis), were most likely prefilled texts. They were not annotated, because it was unclear whether participants used these 'default' motivations on purpose. For example, the most frequent motivation 'my motivation is to use the power of the moustache to have an everlasting impact on the face of mens health' appeared in $104 \mathrm{k}$ profiles. The interrater reliability calculated using Cohen's Kappa was found to be satisfactory to good based on 200 double annotations: injustice $(0.71)$, social identity (0.67) and collective efficacy (0.47) (Landis and Koch, 1977). 


\begin{tabular}{rrrrrrrrrrrrr}
\hline Features & \multicolumn{4}{c}{ Injustice } & \multicolumn{4}{c}{ Social Identity } & \multicolumn{4}{c}{ Collective Efficacy } \\
& $\mathbf{P}$ & $\mathbf{R}$ & $\mathbf{F}_{1}$ & $\mathbf{A U C}$ & $\mathbf{P}$ & $\mathbf{R}$ & $\mathbf{F}_{1}$ & $\mathbf{A U C}$ & $\mathbf{P}$ & $\mathbf{R}$ & $\mathbf{F}_{1}$ & AUC \\
\hline Tokens & 0.813 & 0.789 & 0.801 & 0.833 & 0.768 & 0.792 & 0.779 & 0.790 & 0.595 & 0.656 & 0.624 & 0.708 \\
LDA & 0.789 & 0.802 & 0.795 & 0.829 & $\mathbf{0 . 8 0 9}$ & 0.795 & $\mathbf{0 . 8 0 2}$ & $\mathbf{0 . 8 1 5}$ & 0.514 & $\mathbf{0 . 6 8 8}$ & 0.588 & 0.669 \\
Length & 0.644 & 0.615 & 0.629 & 0.693 & 0.526 & 0.632 & 0.574 & 0.564 & 0.419 & 0.642 & 0.507 & 0.582 \\
Country & 0.422 & 0.559 & 0.481 & 0.522 & 0.495 & 0.493 & 0.494 & 0.524 & 0.373 & 0.498 & 0.426 & 0.523 \\
All & $\mathbf{0 . 8 2 3}$ & $\mathbf{0 . 8 1 0}$ & $\mathbf{0 . 8 1 6}$ & $\mathbf{0 . 8 4 6}$ & 0.777 & $\mathbf{0 . 7 9 9}$ & 0.788 & 0.798 & $\mathbf{0 . 5 9 7}$ & 0.660 & $\mathbf{0 . 6 2 7}$ & $\mathbf{0 . 7 1 0}$ \\
\hline
\end{tabular}

Table 1: Results free-text motivations: precision $(\mathrm{P})$, recall $(\mathrm{R}), \mathrm{F}_{1}$ score and AUC.

From the set of Movember participants with matched Twitter accounts, we annotated a randomly selected subset of 2,108 participants. $21.8 \%$ of the participants had more than one motivation type assigned. We randomly split our dataset into a training and test set (Table 2). We have made our annotations available to other researchers ${ }^{1}$.

\begin{tabular}{rrr}
\hline & Train & Test \\
\hline \# Participants & 1,494 & 614 \\
\% US / UK & $54.8 / 45.2$ & $53.3 / 46.7$ \\
\% Injustice & 37.6 & 40.2 \\
\% Social identity & 48.7 & 46.9 \\
\% Collective efficacy & 36.1 & 35.0 \\
\hline
\end{tabular}

Table 2: Dataset statistics

\section{Classification Experiments}

In this section we present results on automatically identifying the motivations of Movember participants. Because participants may have multiple motivation types, we train binary classification models for each motivation type separately. We use logistic regression with L2 regularization, implemented using the Scikit-learn toolkit (Pedregosa et al., 2011). We report results on the test set using precision, recall, $\mathrm{F}_{1}$ score and the Area Under Curve (AUC) metric. Note that a majority class classifier achieves an AUC of 0.5. Feature development and parameter tuning was done based on cross-validation on the training set. Based on the same set of Movember participants, we explore the use of two different types of data: the provided free-text motivations in Movember profiles (Section 3.1) and tweets of the participants (Section 3.2).

\footnotetext{
${ }^{1}$ http: / / www . dongnguyen.nl/data.html: The identifiers of the Movember and corresponding Twitter accounts, the country, text provided in the Movember profiles, and the annotations.
}

\subsection{Free-text Movember Motivations}

All text is lowercased and tokenized. We explore the following features: 1) Token unigrams and bigrams (frequency values), 2) LDA with 20 topics (Blei et al., 2003) trained on text from all US and UK Movember profiles (with the topic proportions as feature values), 3) Text length, and 4) Country (US $=1, \mathrm{UK}=0$ ) to control for prior motivation distributions in the two countries.

The token features already lead to a high performance, and no notable increase in performance is observed by adding the other features (Table 1). The features with the highest weight are shown in Table 4 . The performance numbers are in line with the obtained inter-annotator agreement. For example, the performance is highest on the injustice category, which also had the highest inter-annotator agreement (and vice versa for collective efficacy).

The lengths of texts alone have predictive power. The texts are short (on average 158.4 characters), but there are markable differences between motivation types. Participants with an injustice motivation write longer motivations: the average length of their texts is 213.74 chars in the training set, compared to 148.24 chars (social identity) and 130.93 chars (collective efficacy).

\begin{tabular}{lll}
\hline Injustice & Social Identity & Collective Efficacy \\
\hline LDA topic $^{a}$ & fun & LDA topic \\
cancer & team & beat \\
friend & moustache & and family \\
lost & mo & change \\
father & grow & yourself \\
had & mustache & all of \\
survivor & LDA topic & awareness \\
prostrate & fuzz & for movember \\
for my & movement & awareness of \\
my & look & last \\
\hline
\end{tabular}

Table 4: Top-weighted features for free-text motivation experiments.

\footnotetext{
${ }^{a}$ topic about family/friends who had cancer

${ }^{b}$ topic about raising funds for research

${ }^{c}$ topic about the Movember campaign
} 


\begin{tabular}{rrrrrrrrrrrrr}
\hline Features & \multicolumn{4}{c}{ Injustice } & \multicolumn{4}{c}{ Social Identity } & \multicolumn{4}{c}{ Collective Efficacy } \\
& $\mathbf{P}$ & $\mathbf{R}$ & $\mathbf{F}_{1}$ & $\mathbf{A U C}$ & $\mathbf{P}$ & $\mathbf{R}$ & $\mathbf{F}_{1}$ & AUC & P & R & F $_{1}$ & AUC \\
\hline 1: Tokens & 0.456 & 0.445 & 0.451 & 0.544 & $\mathbf{0 . 5 2 8}$ & 0.563 & 0.545 & $\mathbf{0 . 5 5 9}$ & $\mathbf{0 . 3 9 4}$ & 0.465 & 0.426 & $\mathbf{0 . 5 4 0}$ \\
2: URLs & 0.421 & 0.304 & 0.353 & 0.511 & 0.469 & $\mathbf{0 . 7 3 6}$ & $\mathbf{0 . 5 7 3}$ & 0.500 & 0.360 & 0.209 & 0.265 & 0.504 \\
3: Mentions & 0.435 & 0.340 & 0.382 & 0.522 & 0.477 & 0.694 & 0.566 & 0.511 & 0.360 & $\mathbf{0 . 7 2 1}$ & $\mathbf{0 . 4 8 0}$ & 0.515 \\
4: Effort & 0.434 & 0.518 & $\mathbf{0 . 4 7 2}$ & 0.532 & 0.489 & 0.531 & 0.509 & 0.520 & 0.363 & 0.498 & 0.420 & 0.513 \\
5: LDA & 0.427 & 0.510 & 0.465 & 0.525 & 0.512 & 0.538 & 0.525 & 0.542 & 0.378 & 0.521 & 0.438 & 0.530 \\
6: Behavior & 0.415 & $\mathbf{0 . 5 2 6}$ & 0.464 & 0.514 & 0.463 & 0.410 & 0.435 & 0.495 & 0.360 & 0.581 & 0.445 & 0.513 \\
1+3+4+5+cntry & $\mathbf{0 . 4 6 3}$ & 0.453 & 0.458 & $\mathbf{0 . 5 5 0}$ & 0.520 & 0.542 & 0.531 & 0.550 & 0.381 & 0.419 & 0.399 & 0.526 \\
\hline
\end{tabular}

Table 3: Results on tweets: precision $(\mathrm{P})$, recall $(\mathrm{R}), \mathrm{F}_{1}$ score and AUC.

\subsection{Tweets}

In this section, we present experiments on identifying the motivations based on Twitter data.

Preprocessing Many of the tweets posted during the time of the campaign are not about the campaign itself. Based on manually selected character sequences ${ }^{2}$, we separate relevant from nonrelevant tweets. The tweets are tokenized using the CMU POS tagger (Owoputi et al., 2013). The average number of tweets per user during the studied period is 109.1 (median: 46.0) and the average number of relevant tweets is 8.0 (median: 4.0 ).

Features We explore the same features as with the free-text motivations and several new features:

- Unigram and bigram tokens: URLs and user mentions are replaced by generic tokens. We only keep tokens used by at least 10 Twitter users and we use their normalized frequency.

- URLs: We extract tokens from URLs by taking the hostname, and paths up to depth 2 (e.g., us.movember.com/team/12345 results in us.movember.com, us.movember.com/team and us.movember.com/team/12345).

- User mentions: The Twitter accounts that are mentioned.

- Effort: Length (\#characters), \#tweets about Movember, \#tweets about Movember/\#total number of tweets.

- LDA with 20 topics (Blei et al., 2003). The model is trained on $1.5 \mathrm{M}$ tweets from 2013 and 2014 about the Movember campaign.

- Country: US=1, UK=0.

- Behavior: Fraction of retweets, tweets that contain a user mention, hashtag, URL, or are a reply. Number of days with a tweet about Movember. Fraction of tweets in each week.
Results The results are reported in Table 3. The URLs and behavior features were excluded from the run with the combined feature set, because their individual results suggest no predictive power (possibly due to the small training set). The results are fairly low and just above the 0.5 AUC value of a random classifier. To test whether the best performing classifiers for each motivation type (based on their AUC scores) are significantly better than a random classifier, we use permutation tests. We permute the labels to break the link with the features and calculate the AUC scores of the classifiers by training and testing on 1000 of such permutations. The best classifiers for the injustice and social identity motivation types are significantly better than random $(p<0.01)$, but the performance of the collective efficacy classifier is only slightly significant $(p<0.05)$.

To understand the low performance numbers, we took a closer look at the task and the data. First, we aimed to get a sense of the difficulty of the task. In a small experiment based on 100 Twitter users from the test set, one of the authors read the tweets and tried to identify the motivations. The results were also low (injustice: 0.488 , social identity: 0.548 , and collective efficacy: 0.590), suggesting that the task in itself is also difficult for humans.

The task is challenging because many users only post a few tweets about the campaign. In our data, 382 users have only one relevant tweet and 1,271 users have 5 relevant tweets or less. Furthermore, many of the tweets posted during the campaign focus on the Movember community (Bravo and Hoffman-Goetz, 2015; Dwi Prasetyo et al., 2015), making it hard to distinguish between the different motivations. For example, instagram.com is among the top three of hostnames for all motivation types. Sometimes participants

\footnotetext{
2 'movember', 'mobro', 'mosista', 'cancer', 'shave', ‘donat', 'tache', 'prostate', 'mo', 'testicular', 'mental' 'men's health'
} 
do explicitely mention their motivation (e.g., 'In honour of my dad, [..], I'm growing a horrible moustache for an incredible cause, \#Movember. Donate here: [LINK]'), but such instances are rare and in general the motivations of participants are much less visible through their tweets.

Social media plays a large role in mediating social relationships and users adapt their behavior to the online communities they are participating in (Danescu-Niculescu-Mizil et al., 2013; Nguyen and Rosé, 2011). This may explain why most participants, regardless of their motivation, emphasize the Movember community and its practices (such as the growing of moustaches) in their tweets. Various studies within the emerging field of Computational Social Science (Lazer et al., 2009) have found that Twitter tends to be a good reflection of society (Lamb et al., 2013; O'Connor et al., 2010). However, our results emphasize that the nature of the used platform influences how humans behave, and that this should be taken into account when interpreting the data. In the case of Movember, Twitter data alone could give a misleading view of the motivations of the campaign's participants.

\section{Motivations and Campaign Behavior}

In this section we present a linear regression analysis $(n=90,484)$ of how motivations affect campaign donations by applying our classifier to all US and UK Movember profile texts. Participants of the Movember campaign can be part of a team. We therefore included actual team membership as a control variable, as we expect that team members increase fundraisers' effort due to peer pressure. In our analysis, we exclude all participants that have predefined motivations $(214,484$ out of the 304,968 profiles), because these may not reflect the actual motivation.

The social identity motivation is the most frequent in both countries, but the countries differ in their distributions regarding the injustice and collective efficacy motivations (Table 5).

\begin{tabular}{lrrr}
\hline & \% Injustice & \% Identity & \% Efficacy \\
\hline UK & 31.0 & 49.7 & 46.1 \\
US & 37.6 & 50.3 & 32.1 \\
\hline
\end{tabular}

Table 5: Motivation distribution based on automatic annotation $(n=90,484)$. Note that participants may have multiple motivations.
On average, US participants donate more than UK participants (Table 6). US campaign participants with an injustice motivation raise significantly (coef $=91.525, p<0.001$ ) more money than participants with a social identity (coef $=$ $-5.479, p=$ not significant) or collective efficacy motivation (coef $=-5.765, p<0.1$ ). Participants that are part of a team raise significantly (coef $=$ 75.849, $p<0.001)$ more money than participants without a team. Similar results were obtained for the UK. Furthermore, participants with a social identity motivation are more often part of a team (UK: $58 \%$ vs. $51 \%$ of the participants without a social identity motivation, US: $80 \%$ vs. $76 \%$ ). The regression analysis reveals that being part of a team has a stronger and more positive effect on the amounts raised than the expression of identity as a motivation in the Movember profiles. Our findings are in line with recent Slacktivism research which proposes that people that express social motivations are reluctant to give more than token support due to a lack of interest in the campaigns cause (van den Broek et al., 2015; Kristofferson et al., 2014). Actual team membership, however, contributes to the effectiveness of online fundraising.

\begin{tabular}{lrrr}
\hline & Injustice & Identity & Efficacy \\
\hline UK (\$) & 203.74 & 128.36 & 123.39 \\
US (\$) & 234.47 & 156.07 & 169.03 \\
\hline
\end{tabular}

Table 6: Average amount raised $(n=90,484)$. British pounds were converted in dollars following the exchange rate in November 2013.

\section{Conclusion}

We explored the task of automatically identifying the motivations of Movember campaign's participants. A classifier based on Movember profile texts performed better than a classifier based on Twitter data, possibly due to the role of Twitter in building social relationships. Based on US and UK Movember data, we found a strong link between motivations and donations, and motivations and team membership. Classification of motivations might help campaign organizers to improve their communication strategies. Our study is limited to the Movember campaign. Future research might diverge to other types of online collective action, such as online petitions and open source communities. We also plan to explore larger datasets and features based on network structures. 
Acknowledgements Thanks to Ariana Need and Anna Priante for helpful discussions and feedback on the article, Movember for support on collecting the Movember accounts and feedback on the article, and Twitter for providing part of the tweets used in this study through the Twitter Data Grant. Thanks also to the anonymous reviewers for their helpful comments. This research was funded in part by the 3TU Federation and the Dutch national project COMMIT. The first author was also supported by the Netherlands Organization for Scientific Research (NWO) grant 640.005.002 (FACT).

\section{References}

Eiji Aramaki, Sachiko Maskawa, and Mizuki Morita. 2011. Twitter catches the flu: Detecting influenza epidemics using Twitter. In Proceedings of the 2011 Conference on Empirical Methods in Natural Language Processing.

David Bamman, Jacob Eisenstein, and Tyler Schnoebelen. 2014. Gender identity and lexical variation in social media. Journal of Sociolinguistics, 18(2):135-160.

Albert Bandura. 2000. Exercise of human agency through collective efficacy. Current Directions in Psychological Science, 9(3):75-78.

René Bekkers and Pamala Wiepking. 2011. A literature review of empirical studies of philanthropy: Eight mechanisms that drive charitable giving. Nonprofit and Voluntary Sector Quarterly, 40(5):924973

David M. Blei, Andrew Y. Ng, and Michael I. Jordan. 2003. Latent dirichlet allocation. Journal of Machine Learning Research, 3:993-1022.

Caroline A. Bravo and Laurie Hoffman-Goetz. 2015. Tweeting about prostate and testicular cancers: Do Twitter conversations and the 2013 Movember canada campaign objectives align? Journal of Cancer Education, pages 1-8.

Brian Cugelman, Mike Thelwall, and Phil Dawes. 2011. Online interventions for social marketing health behavior change campaigns: A meta-analysis of psychological architectures and adherence factors. Journal of medical Internet research, 13(1).

Cristian Danescu-Niculescu-Mizil, Robert West, Dan Jurafsky, Jure Leskovec, and Christopher Potts. 2013. No country for old members: User lifecycle and linguistic change in online communities. In Proceedings of the 22nd international conference on World Wide Web (WWW'13).
Munmun De Choudhury. 2014. Opportunities of social media in health and well-being. XRDS, 21(2):23-27.

Tejas Desai, Afreen Shariff, Aabid Shariff, Mark Kats, Xiangming Fang, Cynthia Christiano, and Maria Ferris. 2012. Tweeting the meeting: An in-depth analysis of Twitter activity at Kidney Week 2011. PLoS ONE, 7(7):e40253.

Nugroho Dwi Prasetyo, Claudia Hauff, Dong Nguyen, Tijs A. van den Broek, and Djoerd Hiemstra. 2015. On the impact of Twitter-based health campaigns: A cross-country analysis of Movember. In The Sixth International Workshop on Health Text Mining and Information Analysis.

Jacob Eisenstein, Brendan O'Connor, Noah A. Smith, and Eric P. Xing. 2010. A latent variable model for geographic lexical variation. In Proceedings of the 2010 Conference on Empirical Methods in Natural Language Processing.

Sherry L. Emery, Glen Szczypka, Eulàlia P. Abril, Yoonsang Kim, and Lisa Vera. 2014. Are you scared yet? Evaluating fear appeal messages in tweets about the tips campaign. Journal of Communication, 64(2):278-295.

Alastair Iain Johnston, Rawi Abdelal, Yoshiko Herrera, and Rose McDermott, editors. 2009. Measuring Identity: A Guide for Social Scientists. Cambridge University Press.

Bert Klandermans. 2004. The demand and supply of participation: Social-psychological correlates of participation in social movements. In The Blackwell Companion to Social Movements, pages 360-379. Blackwell Publishing Ltd.

Kirk Kristofferson, Katherine White, and John Peloza. 2014. The nature of slacktivism: How the social observability of an initial act of token support affects subsequent prosocial action. Journal of Consumer Research, 40(6):1149 - 1166.

Alex Lamb, Michael J. Paul, and Mark Dredze. 2013. Separating fact from fear: Tracking flu infections on Twitter. In Proceedings of the 2013 Conference of the North American Chapter of the Association for Computational Linguistics: Human Language Technologies.

J. Richard Landis and Gary G. Koch. 1977. The measurement of observer agreement for categorical data. Biometrics, 33(1):159-174.

David Lazer, Alex Sandy Pentland, Lada Adamic, Sinan Aral, Albert Laszlo Barabasi, Devon Brewer, Nicholas Christakis, Noshir Contractor, James Fowler, Myron Gutmann, Tony Jebara, Gary King, Michael Macy, Deb Roy, and Marshall Van Alstyne. 2009. Life in the network: the coming age of computational social science. Science (New York, NY), 323(5915):721-723. 
Dong Nguyen and Carolyn P. Rosé. 2011. Language use as a reflection of socialization in online communities. In Proceedings of the Workshop on Language in Social Media (LSM 2011), pages 76-85.

Dong Nguyen, Rilana Gravel, Dolf Trieschnigg, and Theo Meder. 2013. "How old do you think I am? A study of language and age in Twitter. In Proceedings of the Seventh International AAAI Conference on Weblogs and Social Media.

Brendan O'Connor, Ramnath Balasubramanyan, Bryan Routledge, and Noah Smith. 2010. From tweets to polls: Linking text sentiment to public opinion time series. In Proceedings of the Fourth International AAAI Conference on Weblogs and Social Media.

Olutobi Owoputi, Brendan O'Connor, Chris Dyer, Kevin Gimpel, Nathan Schneider, and Noah A. Smith. 2013. Improved part-of-speech tagging for online conversational text with word clusters. In Proceedings of the 2013 Conference of the North American Chapter of the Association for Computational Linguistics: Human Language Technologies.

Fabian Pedregosa, Gaël Varoquaux, Alexandre Gramfort, Vincent Michel, Bertrand Thirion, Olivier Grisel, Mathieu Blondel, Peter Prettenhofer, Ron Weiss, Vincent Dubourg, Jake Vanderplas, Alexandre Passos, David Cournapeau, Matthieu Brucher, Matthieu Perrot, and Édouard Duchesnay. 2011. Scikit-learn: Machine learning in Python. Journal of Machine Learning Research, 12:2825-2830.

Tijs A. van den Broek, David J. Langley, and Michel L. Ehrenhard. 2015. Activist versus slacktivist: A dual path model of online protest mobilization. In Academy of Management Best Paper Proceedings, OCIS Division.

Martijn van Zomeren, Tom Postmes, and Russell Spears. 2008. Toward an integrative social identity model of collective action: A quantitative research synthesis of three socio-psychological perspectives. Psychological bulletin, 134(4):504 -535.

Mackenzie R. Wehner, Mary-Margaret Chren, Melissa L. Shive, Jack S. Resneck Jr, Sherry Pagoto, Andrew B. Seidenberg, and Eleni Linos. 2014. Twitter: an opportunity for public health campaigns. The Lancet, 384(9938):131-132. 